\title{
An algorithm for finding complementary nil dominating set in a fuzzy graph
}

\author{
A.Mohamed Ismayil ${ }^{1}$ and A.Mohamed Ali ${ }^{2}$ \\ ${ }^{1}$ Department of Mathematics Jamal Mohamed College, Trichy, India \\ ${ }^{2}$ Department of Mathematics, G.T.N. Arts College, Dindigul, India
}

Received: 23 July 2016, Accepted: 22 January 2017

Published online: 17 August 2017.

\begin{abstract}
In this paper, effective adjacency matrix of a fuzzy graph is introduced. An algorithm for finding a minimal complementary nil dominating set of fuzzy graph is studied with suitable examples.
\end{abstract}

Keywords: Fuzzy Graph, dominating set, complementary nil dominating set.

\section{Introduction}

In 1965, The theory of fuzzy set was introduced by L.A.Zadeh [12]. In 1975, the notion of fuzzy graph and several fuzzy analogues of graph theoretical concepts such as paths, cycles and connectedness are introduced by Rosenfeld [8]. Bhattacharya [1] has established some connectivity concepts regarding fuzzy cut node and fuzzy bridges. A generalization of intersection graphs to fuzzy intersection graphs are presented by McAlester [3] in 1988. Bhutani [2] introduced M-strong fuzzy graphs. Mordeson [7] introduced the concept of fuzzy line graphs and the basic properties in the year 1993. The concept of domination in fuzzy graphs are introduced by A. Somasundaram and S. Somasundaram [10] in 1998. In 2015, Manjusha and M.S. Sunitha [4] introduced the concept of strong domination in fuzzy graphs using strong arc. Mohamed Ismayil et al. [5] introduced the concept of complementary nil domination in fuzzy graph.

In this paper, the notion of effective adjacency matrix is initiated and also introduced the algorithm for finding the minimal complementary nil dominating set of a fuzzy graph.

\section{Preliminaries}

Definition 1.[8] A fuzzy set $V$ is a mapping $\sigma$ from $V$ to $[0,1]$. A fuzzy graph $G$ is a pair of functions $G=(\sigma, \mu)$ where $\sigma$ is a fuzzy subset of a non-empty set $V$ and $\mu$ is a symmetric fuzzy relation on $\sigma$.(i.e) $\mu(u v) \leq \sigma(u) \wedge \sigma(v)$. The underlying crisp graph of $G=(\sigma, \mu)$ is denoted by $G^{*}=(V, E)$ where $E \subseteq V \times V$.

Definition 2.[8] The scalar cardinality of $S \subseteq V$ is defined by $\sum_{u \in S} \sigma(u)$. The order (denoted by $p$ ) and size (denoted by q) of a fuzzy graph $G$ are the scalar cardinality of $\sigma$ and $\mu$ respectively.

Definition 3.[10] An edge $e=(u, v)$ of a fuzzy graph is called an effective edge if $\mu(u, v)=\sigma(u) \wedge \sigma(v)$. 
Definition 4.[10] Let $G=(\sigma, \mu)$ be a fuzzy graph and let $u, v \in V$. If $\mu(u, v)=\sigma(u) \wedge \sigma(v)$ then $u$ dominates $v$ (or $v$ is dominated by $u$ ) in $G$. A subset $D$ of $V$ is called a dominating set in $G$ if for every $v \notin D$ there exist $u \in D$ such that $u$ dominates $v$. The minimum scalar cardinality taken over all dominating set is called domination number and is denoted by the symbol $\gamma$. The maximum scalar cardinality of a minimal dominating set is called upper domination number and is denoted by the symbol $\Gamma$.

Definition 5.[10] A set $S \subset V$ in a fuzzy graph $G=(\sigma, \mu)$ is said to be independent set if no two vertices of $S$ are adjacent.

Definition 6.[5] Let $G=(\sigma, \mu)$ be a fuzzy graph on $V$. A set $S \subset V$ is said to be a complementary nil dominating set (or simply called cnd-set) of a fuzzy graph $G$ if $S$ is a dominating set and its complement $V-S$ is not a dominating set.

Definition 7.[5] A cnd-set $S$ of a fuzzy graph $G=(\sigma, \mu)$ is called a minimal cnd-set if there is no cnd-set $S^{\prime}$ such that $S^{\prime} \subset S$.

Definition 8.[5] A cnd-set $S$ of a fuzzy graph $G=(\sigma, \mu)$ is called a minimum cnd-set if there is no cnd-set $S^{\prime}$ such that $\left|S^{\prime}\right|<|S|$. The minimum scalar cardinality taken over all cnd-set is called a complementary nil domination number and is denoted by the symbol $\gamma_{c n d}$, the corresponding minimum cnd-set is denoted by $\gamma_{\text {cnd-set. }}$.

\section{Effective adjacency matrix}

Definition 9. Let $G$ be a fuzzy graph on underlying graph $G^{*}$ with $n$ vertices. Then the effective adjacency matrix of $G$ is a $n \times n$ matrix whose entries are defined by

$$
a_{i j}=\left\{\begin{array}{l}
1 \text { if either } i=j \text { or }\left(v_{i}, v_{j}\right) \text { is an effective edge } \\
0 \text { otherwise }
\end{array}\right.
$$

and it is denoted by $\operatorname{EAM}(A)$.

Example 1.Consider the fuzzy graph as given in Figure 1.

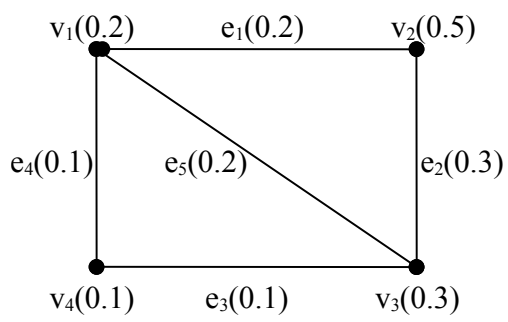

Fig. 1: Illustration of the Algorithm

The effective adjacency matrix $\operatorname{EAM}(A)=\left(\begin{array}{ccccc}\left(v_{1}\right) & \left(v_{2}\right) & \left(v_{3}\right) & \left(v_{4}\right) \\ \left(v_{1}\right) & 1 & 1 & 1 & 1 \\ \left(v_{2}\right) & 1 & 1 & 1 & 0 \\ \left(v_{3}\right) & 1 & 1 & 1 & 1 \\ \left(v_{4}\right) & 1 & 0 & 1 & 1\end{array}\right)$. 


\section{Algorithm for finding complementary nil dominating set in a fuzzy graph}

Let us assume that $G=(V, E)$ be a fuzzy graph without isolated vertex but not complete.

Step 1:

Write the effective adjacency matrix $E A M(A)$ of a given fuzzy graph.

$\operatorname{EAM}(A)=\operatorname{EAM}_{o}(A)$

Step 2:

Calculate the number of 1 's occurred in each row of $\operatorname{EAM}(A)$.

Step 3:

$E A M(A)=R(A)$. If $n$ rows has exactly two 1 's, then choose the vertex $v_{i}$ with minimum membership value among the vertices. (If more than one vertices has same membership values then choose a vertex arbitrarily, goto step 5. Otherwise goto step 4

\section{Step 4:}

In $R(A)$, Choose the row with maximum number of 1's.(say $i^{\text {th }}$ row)

If more than one row has an equal number of 1's, then choose a vertex with minimum membership value.(If more than one vertex has same membership value then choose arbitrarily one vertex).

\section{Step 5:}

Put $v_{i}$ in $D$.

Step 6:

Delete the row and column corresponding to $v_{i}$ in $R(A)$.

Step 7:

Calculate $D_{1}=V-D$.

Delete the row corresponding to $v_{i}$ in $\operatorname{EAM}(A)$.

Step 8:

Calculate the column total of $\operatorname{EAM}(A)$.If any one of the total is zero, then $D_{1}=V-D$ is not a dominating set. Goto step 9 Otherwise $D_{1}=V-D$ is a dominating set, then goto step 4

\section{Step 9: [Check $D$ is a dominating set or not]}

Delete all the rows corresponding to the vertices of $D_{1}$ in $E A M_{o}(A)$

Calculate the column total of $\operatorname{EAM}_{o}(A)$.

If all the column totals are non-zero, then goto step 10. Otherwise goto step 4 .

\section{Step 10:}

$D$ is a dominating set and also it is a minimal complementary nil dominating set.

Example 2.Consider the fuzzy graph as given in Figure 2.

Step: 1

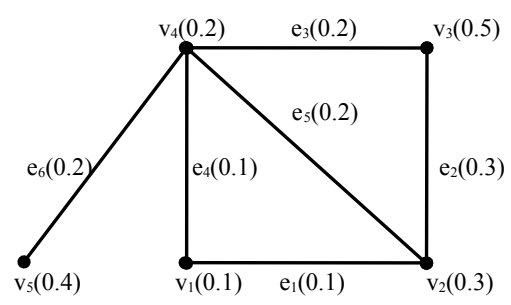

Fig. 2: Illustration of the Algorithm 
The effective adjacency matrix $\operatorname{EAM}(A)=\left(\begin{array}{ccccccc}\left(v_{1}\right) & \left(v_{2}\right) & \left(v_{3}\right) & \left(v_{4}\right) & \left(v_{5}\right) \\ \left(v_{1}\right) & 1 & 1 & 0 & 1 & 0 \\ \left(v_{2}\right) & 1 & 1 & 1 & 1 & 0 \\ \left(v_{3}\right) & 0 & 1 & 1 & 1 & 0 \\ \left(v_{4}\right) & 1 & 1 & 1 & 1 & 1 \\ \left(v_{5}\right) & 0 & 0 & 0 & 1 & 1\end{array}\right) \operatorname{EAM}(A)=\operatorname{EAM}_{o}(A)$

\section{Step: 2}

The number of 1's occurred in row 1, row 2, row 3, row 4 and row 5 are 3, 4, 3, 4 and 2 respectively.

Step: 3

$E A M(A)=R(A)$ The row 5 has exactly two 1's. Choose $v_{5}$.

\section{Step: 5}

Put $v_{5}$ in $D$.

\section{Step: 6}

Delete a row and a column corresponding to $v_{5}$ in $R(A)$.

\section{Step: 7}

Calculate $D_{1}=V-D=\left\{v_{1}, v_{2}, v_{3}, v_{4}\right\}$.

Delete the row corresponding to $v_{5}$ in $\operatorname{EAM}(A)$.

$$
\operatorname{EAM}(A)=\left(\begin{array}{cccccc}
\left(v_{1}\right) & \left(v_{2}\right) & \left(v_{3}\right) & \left(v_{4}\right) & \left(v_{5}\right) \\
\left(v_{1}\right) & 1 & 1 & 0 & 1 & 0 \\
\left(v_{2}\right) & 1 & 1 & 1 & 1 & 0 \\
\left(v_{3}\right) & 0 & 1 & 1 & 1 & 0 \\
\left(v_{4}\right) & 1 & 1 & 1 & 1 & 1
\end{array}\right)
$$

Step: 8

$$
\operatorname{EAM}(A)=\left(\begin{array}{cccccc} 
& \left(v_{1}\right) & \left(v_{2}\right) & \left(v_{3}\right) & \left(v_{4}\right) & \left(v_{5}\right) \\
\left(v_{1}\right) & 1 & 1 & 0 & 1 & 0 \\
\left(v_{2}\right) & 1 & 1 & 1 & 1 & 0 \\
\left(v_{3}\right) & 0 & 1 & 1 & 1 & 0 \\
\left(v_{4}\right) & 1 & 1 & 1 & 1 & 1 \\
& --- & -- & -- & -- & -- \\
& 3 & 4 & 3 & 4 & 1
\end{array}\right)
$$

The column totals of $\operatorname{EAM}(A)$ are 3, 4, 3, 4 and 1. Hence all the column totals are non-zero. Goto step 4.

Step: 4

$$
R(A)=\left(\begin{array}{ccccc}
\left(v_{1}\right) & \left(v_{2}\right) & \left(v_{3}\right) & \left(v_{4}\right) \\
\left(v_{1}\right) & 1 & 1 & 0 & 1 \\
\left(v_{2}\right) & 1 & 1 & 1 & 1 \\
\left(v_{3}\right) & 0 & 1 & 1 & 1 \\
\left(v_{4}\right) & 1 & 1 & 1 & 1
\end{array}\right)
$$

The number of 1's occurred in row 1, row 2, row 3 and row 4 are 3, 4, 3 and 4 respectively. The rows 2 and 4 has equal of no of 1 's. Then minimum weight of $v_{2}$ and $v_{4}$ is $v_{4}$. Choose $v_{4}$.

Step: 5

Put $v_{4}$ in $D$.

Step: 6

Delete the row and column corresponding to $v_{4}$ in $R(A)$. 


$$
R(A)=\left(\begin{array}{cccc}
\left(v_{1}\right) & \left(v_{2}\right) & \left(v_{3}\right) \\
\left(v_{1}\right) & 1 & 1 & 0 \\
\left(v_{2}\right) & 1 & 1 & 1 \\
\left(v_{3}\right) & 0 & 1 & 1
\end{array}\right)
$$

\section{Step:7}

Calculate $D_{1}=V-D=\left\{v_{1}, v_{2}, v_{3}\right\}$.

Delete the row corresponding to $v_{4}$ in $\operatorname{EAM}(A)$.

\section{Step:8}

$$
\operatorname{EAM}(A)=\left(\begin{array}{cccccc} 
& \left(v_{1}\right) & \left(v_{2}\right) & \left(v_{3}\right) & \left(v_{4}\right) & \left(v_{5}\right) \\
\left(v_{1}\right) & 1 & 1 & 0 & 1 & 0 \\
\left(v_{2}\right) & 1 & 1 & 1 & 1 & 0 \\
\left(v_{3}\right) & 0 & 1 & 1 & 1 & 0 \\
& --- & -- & -- & -- & -- \\
2 & 3 & 2 & 3 & 0
\end{array}\right)
$$

The column totals of $\operatorname{EAM}(A)$ are 2, 3, 2, 3 and 0 . Hence one of the column total is zero. Goto step 9.

\section{Step:9 Check $D$ is a dominating set or not}

Delete all the rows corresponding to the vertices of $D_{1}$ in $E A M_{o}(A)$.

Calculate the column total of $\operatorname{EAM}_{o}(A)$.

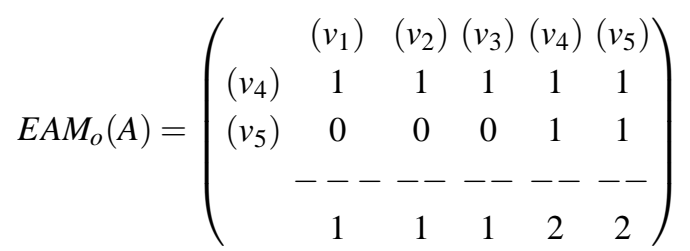

All the column totals are non-zero. goto step 10.

\section{Step:10}

$D=\left\{v_{4}, v_{5}\right\}$ is a dominating set and also it is a minimal complementary nil dominating set.

Example 3.Consider a fuzzy graph $G=(\sigma, \mu)$ given in FIGURE 3, where $\sigma=\left\{u_{1} / 0.3, u_{2} / 0.4, u_{3} / 0.6, u_{4} / 0.2, u_{5} / 0.8, u_{6} / 0.7\right\}$ and $\mu=\left\{\left(u_{1}, u_{2}\right) / 0.3\right.$, $\left.\left(u_{2}, u_{3}\right) / 0.4,\left(u_{3}, u_{4}\right) / 0.2,\left(u_{4}, u_{5}\right) / 0.2,\left(u_{5}, u_{6}\right) / 0.7,\left(u_{6}, u_{1}\right) / 0.3,\left(u_{3}, u_{6}\right) / 0.4\right\}$, $S=\left\{u_{1}, u_{2}, u_{3}, u_{4}\right\}$ - is $\gamma_{c n d}$-set.

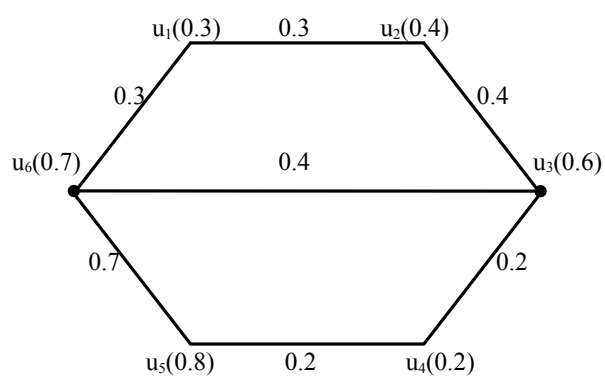

Fig. 3: Fuzzy Graph 


\section{Competing interests}

The authors declare that they have no competing interests.

\section{Authors' contributions}

All authors have contributed to all parts of the article. All authors read and approved the final manuscript.

\section{References}

[1] Bhattacharya, Some remarks on fuzzy graphs, Pattern Recognition Letter 6(1987) 297-302.

[2] K. R. Bhutani, On M-strong fuzzy graphs, Information Sciences 155(2003) 103-109.

[3] M. L. N. McAlester, Fuzzy intersection graphs, Comp.Math. Appl. 15(10) 871-886.

[4] O.T. Manjusha, M.S.Sunitha, Strong Domination in Fuzzy Graphs, Fuzzy Information and Engineering Volume 7, Issue 3, September 2015, Pages 369 - 377.

[5] A. Mohamed Ismayil and S. Ismail Mohideen, Complementary nil domination in fuzzy graphs,Ann. Fuzzy Math. Inform. 8(5) (2014) 785-792.

[6] A. Mohamed Ismayil and A.Mohamed Ali, Complementary nil domination in intuitionistic fuzzy graph using effective edges, Ann. Fuzzy Math. Inform., 10(5) (2015), pp.765-774.

[7] J. N. Mordeson, Fuzzy line graphs, Pattern Recognition Letter 14 (1993) 381-384.

[8] A. Rosenfeld, Fuzzy graphs, in: L.A. Zedeh, K.S. Fu, K. Tanaka, M. Shimura(Eds.), Fuzzy sets and Their Applications to Cognitive and Decision Processes, Academic Press, NewYork (1975) 77-95.

[9] S. I. Mohideen and A. M. Ismayil, Domination in fuzzy graph: A new approach, Int. J. Comput.Sc. Math. 2 (3) (2010) $101-107$.

[10] A. Somasundaram and S. Somasundaram, Domination in fuzzy graph-I, Patter Recognit. Lett. 19(9) (1998) 787-791.

[11] T. Tamizh Chelvam and S. Robinson Chellathurai, Complementary nil domination number of a graph, Tamkang Journal of Mathematics, Vol. 40, No.2(2009), 165-172.

[12] L.A.Zadeh, Fuzzy Sets, Inform Control.,8(1965), 338-353. 